\title{
Generation and detection of thermoelastic waves in metals by a photothermal mirror method
}

\author{
O. A. Capeloto, ${ }^{1}$ V. S. Zanuto, ${ }^{1}$ G. V. B. Lukasievicz, ${ }^{2}$ L. C. Malacarne,${ }^{1}$ S. E. Bialkowski, ${ }^{3}$ \\ T. Požar, ${ }^{4}$ and N. G. C. Astrath ${ }^{1, a)}$ \\ ${ }^{1}$ Departamento de Física, Universidade Estadual de Maringá, Maringá, PR 87020-900, Brazil \\ ${ }^{2}$ Departamento de Física, Universidade Tecnológica Federal do Paraná, Medianeira, PR 85884-000, Brazil \\ ${ }^{3}$ Department of Chemistry and Biochemistry, Utah State University, Logan, Utah 84322, USA \\ ${ }^{4}$ Faculty of Mechanical Engineering, University of Ljubljana, Ljubljana 1000, Slovenia
}

(Received 21 September 2016; accepted 26 October 2016; published online 9 November 2016)

\begin{abstract}
We investigate the thermoelastic waves launched by a localized heat deposition. Pulsed laser excitation is used to generate mechanical perturbations in metals that are detected using the photothermal mirror method. This method detects the wavefront distortion of the probe beam reflected from the perturbed sample surface. Nanometer scale expansion of the material is induced just under the irradiated surface releasing transient thermoelastic waves of much smaller amplitudes on the surface. Numerical predictions and the experimental results are in a good agreement and represent both the thermal diffusion of the large amplitude, long-lasting outward bulge, and the released elastic waves. Published by AIP Publishing. [http://dx.doi.org/10.1063/1.4967530]
\end{abstract}

The interaction between the light pulse and the surface of an opaque solid may generate elastic waves by heating the material (thermoelastic effect) or by the momentum transfer from the light to the solid (radiation pressure). ${ }^{1}$ In this energy transfer process, part of the energy is absorbed in a thin layer of the surface of the solid and then converted into heat. ${ }^{2-4}$ The rapid deposition of the thermal energy acts as the source of mechanical waves in the material. The thermal expansion of the solid induces various types of mechanical disturbances. ${ }^{5-9}$ Within the bulk of the material, longitudinal (P-waves) and shear waves (S-waves) radiate spherically away from this source. They are accompanied by the surface-bound Rayleigh waves (R-waves) and the Machcone shaped head waves (H-waves). ${ }^{3,4}$ At high power density levels, the ablation effect may occur modifying the state of the surface. Below the ablation threshold, at moderate power densities, thermoelastic waves dominate over radiation pressure induced waves. ${ }^{10}$

Detection of light-induced elastic waves is feasible with many different experimental approaches. ${ }^{3-15}$ Bulk and surface waves can be detected utilizing capacitive, piezoelectric, electromagnetic, and optical transduction mechanisms. ${ }^{16}$ The possibility of remote generation and stand-off detection of elastic waves using optical probes makes photothermal methods attractive for material characterization ${ }^{15}$ and non-destructive testing. ${ }^{2-4}$ A number of pump-probe methods have been employed to detect mechanical perturbations in solids using either interferometric schemes, photothermal beam deflection, or by analyzing diffraction wavefront distortions. As opposed to the capacitive, electromagnetic, or piezoelectric sensors, the above-mentioned optical methods can be used to detect elastic waves created just underneath the pulse excitation area. ${ }^{9,15,17,18}$ The ability of probing the region of the largest temperature variation and mechanical deformation makes them an attractive complementary approach.

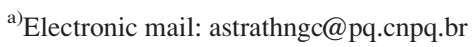

Mechanical displacement can be detected by analyzing the focusing or defocusing of the probe beam reflected from the sample surface in the far field region. ${ }^{11-14,19,20}$ This method is referred to as photothermal mirror (PTM) spectrometry and has been recently introduced under pulsed Gaussian laser excitations for the measurement of thermal diffusivity and thermo-optical properties of semi-transparent and opaque solids. ${ }^{12,13}$ Using a slightly different experimental approach, this method was also employed to measure the surface deformation induced by radiation pressure at air-liquid interfaces induced by continuous and pulsed laser excitation. ${ }^{19,20}$ Here we show, for pulsed laser excitation in metals, that the launched elastic waves propagate on the surface and within the solids generating convoluted photothermal mirror signals following the thermo-mechanical properties of the sample. The elastic waves are predicted by the numerical solution of the thermoelastic equation and have properties closely related to the familiar elastic waves created by an isotropic point-expansion source on the surface of solids.

The measurements of elastic waves are performed by exciting an opaque metal using a laser pulse with a Gaussian transverse spatial distribution $\left(\mathrm{TEM}_{00}\right)$. The beam is directed normal to the surface of the sample, propagating along the $z$ axis, as illustrated in Fig. 1. Part of the laser energy is absorbed at a skin depth of the sample surface and converted into heat, and part of the energy is reflected. By conduction, heat is transferred to the surrounding fluid (air). Since the problem is circular symmetric, the fields depend only on the normal $z$ - and radial $r$-coordinates. The temperature elevations, $T_{j}(r, z, t)$, in the sample $(j=s)$ and in the surrounding fluid $(j=f)$ follow separate heat diffusion equations ${ }^{13}$

$$
\frac{\partial T_{j}(r, z, t)}{\partial t}-D_{j} \nabla^{2} T_{j}(r, z, t)=\frac{\phi_{j}}{\rho_{j} C_{p j}} e^{-2 r^{2} / w_{e}^{2}} \delta(z) f(t) .
$$

$D_{j}=k_{j} / \rho_{j} C_{p j}$ is the thermal diffusivity, $k_{j}$ is the thermal conductivity, $\rho_{j}$ is the mass density, $C_{p j}$ is the specific heat, $t$ is the time, $R$ is the reflectivity at the excitation wavelength, 


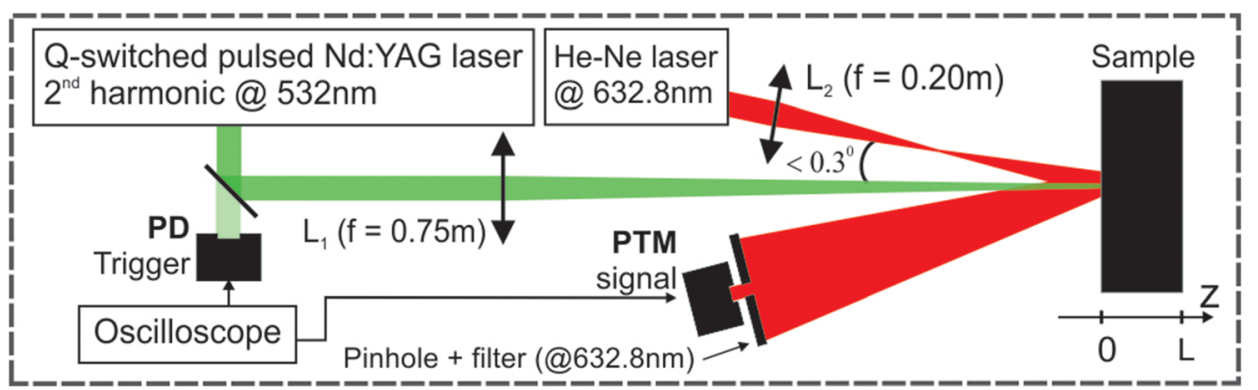

FIG. 1. Experimental diagram for the PTM measurements. The pump and probe beam are focused by biconvex lenses of focal lengths $f$. Experimental parameters are $w_{e}=130 \mu \mathrm{m}, w_{p}=$ $580 \mu \mathrm{m}, z_{c}=3.3 \mathrm{~cm}$, and $z_{1}=25.6 \mathrm{~cm}$. The temperature of the samples was $298 \mathrm{~K}$. and $w_{e}$ is the radius of the excitation beam on the surface of the sample. Radiation losses are neglected. The temporal distribution of the laser pulse is represented by $f(t)$ $=t_{0}^{-1} e^{-(t-\xi)^{2} / \tau^{2}}$, with the time of maximum irradiance and width given by $\xi$ and $\tau$, respectively. Since the penetration depth is less than $5 \mathrm{~nm}$ for the samples investigated in this work, the deposition of thermal energy by light absorption is assumed to occur only at the surface of the sample. This is represented by a Dirac delta function $\delta(z)$. The amplitude of the heat source is $\phi_{s}=4 Q_{0}(1-R) /\left(\pi w_{e}^{2}\right)$ and there is no absorption in the fluid, i.e., $\phi_{f}=0$. The pulse energy is $Q_{0}$, and $t_{0}=\tau \sqrt{\pi}[1+\operatorname{erf}(\xi / \tau)] / 2$ is the normalization parameter.

Once the temperature field is obtained for any time by solving Eq. (1), it is then used as the source to compute the induced bulk and surface waves by solving the thermoelastic equation of motion with the appropriate quiescent initial and boundary conditions. The displacement field $\mathbf{u} \equiv \vec{u}(r, z, t)$ of the solid is given by the solution of ${ }^{13}$

$$
(\lambda+2 \mu) \nabla^{2} \mathbf{u}+(\lambda+\mu) \nabla(\nabla \cdot \mathbf{u})=\gamma \nabla T_{s}(r, z, t)+\rho \frac{\partial^{2} \mathbf{u}}{\partial t^{2}},
$$

where $\gamma=(3 \lambda+2 \mu) \alpha_{T} . \lambda=E \nu /[(1+\nu)(1-2 \nu)]$ and $\mu=$ $E / 2(1+\nu)$ are the Lame's constants, $E$ is the Young's modulus, and $\nu$ is the Poisson's ratio. Due to a large impedance mismatch between the air and the solid, we neglect the mechanical coupling with air. The last term on the righthand side of Eq. (2) represents the inertia term yielding elastic wave motion.

The axial surface deformation given by the solution of Eq. (2), $u_{z}(r, z=0, t)$, is measured by analyzing the on-axis intensity change of the central portion of the probe beam reflected from the sample surface at the far field photodetector. ${ }^{19}$ On the surface under investigation, the centers of both the pump and the probe beams overlap. The circularly symmetric elastic waves created at the surface act as the dynamic optical elements to the wavefront of the probe beam increasing or diminishing its amplitude signal passing the pinhole at the detector plane. $u_{z}(r, 0, t)$ produces a phase shift to the reflected portion of the probe beam as $\Phi(r, t)=\left(4 \pi / \lambda_{p}\right) u_{z}(r, 0, t)$, where $\lambda_{p}$ is the probe beam wavelength. The optical power of the reflected probe beam passing the pinhole varies as ${ }^{11}$

$$
S(t)=\left|\int_{0}^{\infty} \frac{2 r}{w_{p}^{2}} \exp \left[-\left(1+i \frac{z_{1}}{z_{c}}\right) \frac{r^{2}}{w_{p}^{2}}-i \Phi(r, t)\right] \mathrm{d} r\right|^{2},
$$

where $z_{c}$ is the confocal distance of the probe beam, $z_{1}$ is the distance from the probe beam waist to the sample, and $w_{p}$ is the radius of the probe beam at the sample surface. Eq. (3) can be evaluated numerically using the calculated surface deformation field $u_{z}(r, 0, t)$. Additional phase shift to the probe beam as a consequence of the thermal lens effect created in the surrounding air by heat coupling to the sample, or the influence of the temperature dependence of the physical properties of the samples on the signal, can be safely neglected for the experiments performed in this study.

The pump-probe configuration of the photothermal mirror experiment is schematically shown in Fig. 1. The pulsed laser (Q-switched Nd:YAG laser; Quantel, Brilliant B at $532 \mathrm{~nm}, \xi=45 \mathrm{~ns}$ and $\tau=15 \mathrm{~ns}$ ) is arranged almost collinear to the probe laser beam (He-Ne laser; Newport at $632.8 \mathrm{~nm}$ ). The intensity variation of the probe beam center after reflection was detected by a pinhole-laser line filter-photodetector (Newport, Model 818BB-22) assembly in the far field. A digital oscilloscope (Tektronix, Model DPO 4102B) triggered by the photodiode (Newport, Model 818BB-22) recorded the data. Due to the wide frequency bandwidth (DC to $200 \mathrm{MHz}$ ) of this homodyne detection method, both the fast transients, such as the propagation elastic waves, and the slower thermal-diffusion-driven changes of the surface displacement can be monitored by a single stand-off detector.

Experimental PTM signals were generated on copper and aluminum disks of $1.6 \mathrm{~cm}$ in diameter and thickness $L$. The surface of the samples was polished to a mirrored surface and irradiated with focussed laser pulses with energies varying from $10 \mu \mathrm{J}$ to $50 \mu \mathrm{J}$. The PTM signals presented in Fig. 2 are averaged over 100 transients at a repetition frequency of $10 \mathrm{~Hz}$. The time interval between consecutive events is long enough for the sample to return to its unperturbed state. The transients show the intensity change of the central portion of the continuous probe beam reflected from the sample surface at the far field sensor. The energies and experimental parameters are listed in the figures.

The PTM signal is a complex convolution of the probe beam wavefront distortion caused by the surface displacement. Its shape can be quantitatively assessed by comparing the experiments to the theoretical signal given by Eq. (3). The heat diffusion and the thermoelastic equations were numerically evaluated by the finite element analysis (FEA) method using the software Comsol Multiphysics 4.3b. The model was built in the 2D axisymmetric geometry and employed to solve Eqs. (1) and (2) with a realistic sample geometry assuming heat coupling from metal to air with appropriated boundary and initial conditions, and the physical 


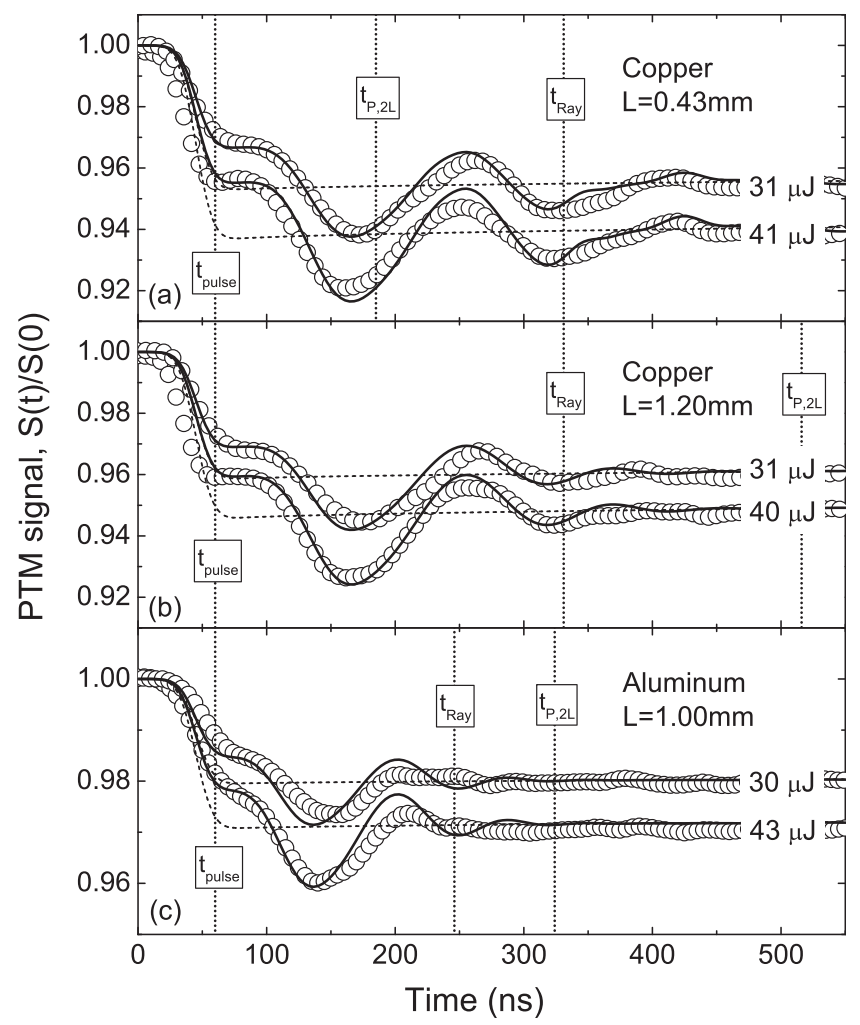

FIG. 2. Measured (circles) and FEA calculated (lines) PTM signal for ((a) and (b)) copper and (c) aluminum metals with different thicknesses $L$. Dashed lines represent the FEA calculated signal without the inertia term in Eq. (2). Vertical lines denote: the end of illumination $\left(t_{\text {pulse }}\right)$, the return time of flight of the P-wave across the thickness of the plate $\left(t_{\mathrm{P}, 2 \mathrm{~L}}\right)$, and time at which the Rayleigh wave leaves the probed area $\left(t_{\text {Ray }}\right)$. Physical properties are listed in supplementary material Table SI.

properties of the samples. ${ }^{21}$ Numerical results for $u_{z}(r, 0, t)$ are then used to calculate the probe beam wavefront distortion and the PTM signal, Eq. (3). Continuous lines in Fig. 2 show the calculated PTM signals. The numerical predictions are in good agreement with measurements. By comparison, the calculated PTM signal without the inertia term in Eq. (2), known as the quasistatic approximation, is also presented with dashed lines.

The laser pulse generates various thermoelastic waves that can be seen in Fig. 3 by assessing the FEA calculated radial $v_{r}(r, z, t)$ and axial $v_{z}(r, z, t)$ components of the particle velocity field, with $v_{j}(r, z, t)=\partial u_{j}(r, z, t) / \partial t$. The velocity fields represent the wavefronts at successive times after the interaction. Soon after the pulse is absorbed, mechanical waves are indistinguishable in the core of the sample. In the far field, just before the P-wave reaches the other side of plate, i.e., at $240 \mathrm{~ns}$ for the case presented in Fig. 3, separate wave-types clearly decouple so that all elastic waves can be identified. Their propagation velocities $\left(c_{\mathrm{P}}, c_{\mathrm{S}}, c_{\mathrm{R}}\right)$ are listed in supplementary material, Table SI. Before the time the P-wave reflects back to the illuminated surface at $t_{P, 2 L}=$ $2 L / c_{P}$ (Fig. 2), only the surface propagating P-, S-, and Rwaves distort the probing beam. After this time, the multiply reflected $(2 \mathrm{P}, 2 \mathrm{~S}, 4 \mathrm{P}, \ldots)$ and mode converted bulk waves (PS, 3PS, ...) arrive to the front surface additionally affecting the shape of the thermal mirror. $t_{\text {Ray }}$ in Fig. 2 labels the time $t_{\text {Ray }}=\left(w_{e}+w_{p}\right) / c_{\mathrm{R}}$ when the slowest propagating surface waves, the R-waves originating from the far edge of

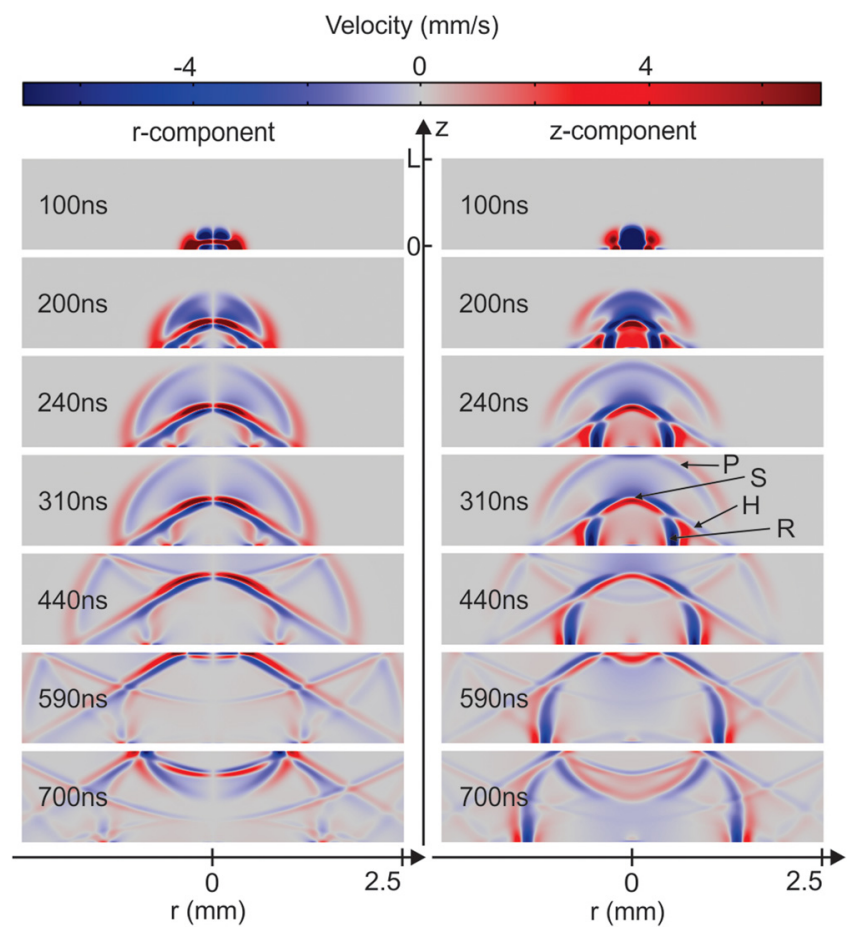

FIG. 3. Simulated velocity field time frames, $v_{r}(r, z, t)$ and $v_{z}(r, z, t)$, for copper with $L=1.20 \mathrm{~mm}$.

the interaction zone, leave the central part of the probing area. $t_{\text {pulse }}$ marks the end of illumination.

One can see from Fig. 2 that as long as the surface waves propagate within the probed area, the modeled PTM signals conform to the measured ones if the inertial term is included and largely deviate when this term is excluded. When the surface waves exit the probing spot, the inclusion of the inertial term gives almost identical results as if it was not taken into account. Further, the bulk waves reflected from the opposite surface insignificantly affect the probing surface because their wavefronts are nearly parallel with the surface and cause a large curvature displacement of the whole probing area.

Figure 4 shows the time dependence of the modeled (FEA calculated) surface displacement, $u_{z}(r, 0, t)$, at different distances $r$ from the epicenter. At the epicenter, $r=0$,

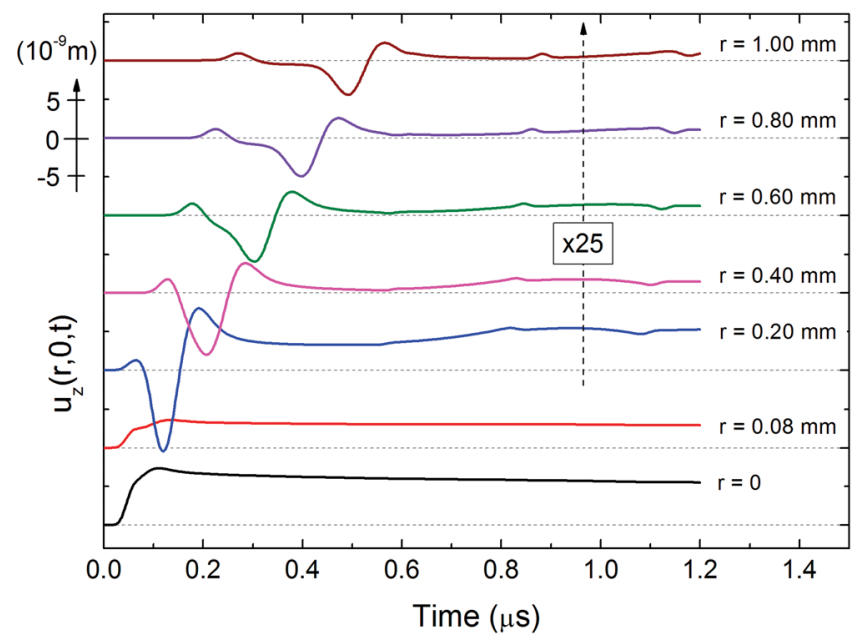

FIG. 4. Simulated surface displacement for copper with $L=1.20 \mathrm{~mm}$ at different distances $r$ from the epicenter. Positive $u_{z}(r, 0, t)$ means outward displacement. 
Surface displacement, $u_{z}(r, 0, t),(n m)$

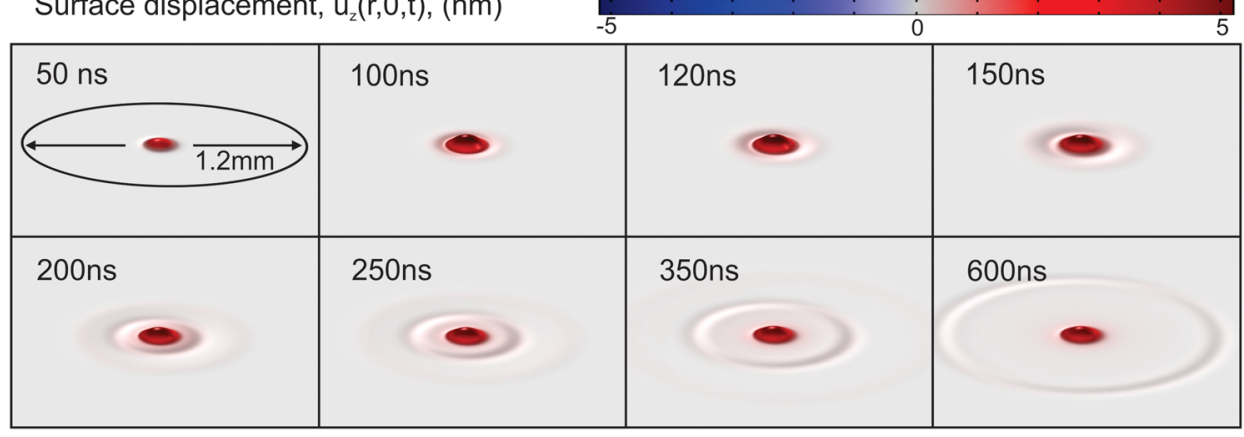

FIG. 5. Simulated time evolution of the surface displacement of copper $(L=1.20 \mathrm{~mm})$. heat is locally deposited by the pulse on the surface and thermal expansion governs the displacement of the surface. ${ }^{9}$ Large displacements of $7 \mathrm{~nm}$ are created around the vicinity of the excitation area. Elastic waves are resolved away from the epicenter as shown for $r>200 \mu \mathrm{m}$. For example, at $r=1.00 \mathrm{~mm}$, the surface skimming $\mathrm{P}$-wave arrives at about $250 \mathrm{~ns}$. The R-wave follows between $400 \mathrm{~ns}$ and $600 \mathrm{~ns}$, and then the reflected bulk waves arrive. The outward P-wave displacement followed by the inward phase of the R-wave is a clear signature of elastic waves generated by thermal expansion. ${ }^{3,4}$ If the initial phase of the R-wave was outward, the dominating source would be either ablation or radiation pressure, while the latter never dominates in metals.

The probe beam reflected from the sample senses the complex surface deformation history displayed in Fig. 5. The illuminated area experiences a large, long-lasting swelling that is responsible for the overall decrease of the PTM signal. Superimposed on this dominating effect, different radial annuli of the probed beam can be either focused (positive displacement slopes of the surface propagating waves) or dispersed (negative slopes). This in turn leads to the increase or decrease of the power that passes through the pinhole placed in front of the detector.

We have shown that the applicability of the photothermal mirror method can be extended to visualize laser induced thermoelastic waves generated locally and propagating within metals. Heat deposition by the pulse launches bulk and surface waves and generates large surface swelling of the sample under the excitation area. Numerical solutions of the coupled heat diffusion and thermoelastic equations predict the detected photothermal mirror signal for copper and aluminum samples. Velocity field calculations show the moving wavefronts of various types of elastic waves inside the samples. Wavefront distribution is complex short after the pulse and decouples further in time evidencing internal reflection at the facets of the samples. The surface propagating waves, especially the Rayleigh wave, significantly affect the photothermal mirror signal, while the multiply reflected bulk waves only gently perturb the probed surface. The detection of elastic waves on top of the large bulge enables characterization of thermal and also elastic properties of opaque solids.
See supplementary material for the physical properties of the samples.

This work was supported by CAPES, CNPq, and Fundação Araucária, and partially conducted as a part of the Optodynamics program (P2-0392), in duration from 20151-1 to 2019-12-31, financed by the Slovenian Research Agency.

${ }^{1}$ T. Požar and J. Možina, Phys. Rev. Lett. 111, 185501 (2013).

${ }^{2}$ V. E. Gusev and A. A. Karabutov, Laser Optoacoustics (American Institute of Physics, New York, 1993).

${ }^{3}$ D. Royer and E. Dieulesaint, Elastic Waves in Solids II (Springer, Berlin, 2000).

${ }^{4}$ C. B. Scruby and L. E. Drain, Laser Ultrasonics, Techniques and Applications (Adam Hilger, New York, 1990).

${ }^{5}$ S. J. Davies, C. Edwards, G. S. Taylor, and S. B. Palmer, J. Phys. D 26, 329 (1993).

${ }^{6}$ D. A. Hutchins, Can. J. Phys. 64, 1247 (1986).

${ }^{7}$ F. A. McDonald, Appl. Phys. Lett. 54, 1504 (1989).

${ }^{8}$ R. I. Dewhurst, D. A. Hutchins, S. B. Palmer, and C. B. Scruby, J. Appl. Phys. 53, 4064 (1982).

${ }^{9}$ J. B. Spicer and D. H. Hurley, Appl. Phys. Lett. 68, 3561 (1996).

${ }^{10}$ T. Požar, A. Babnik, and J. Možina, Opt. Express 23, 7978 (2015).

${ }^{11}$ L. C. Malacarne, F. Sato, P. R. B. Pedreira, A. C. Bento, R. S. Mendes, M. L. Baesso, N. G. C. Astrath, and J. Shen, Appl. Phys. Lett. 92, 131903 (2008).

${ }^{12}$ G. V. B. Lukasievicz, N. G. C. Astrath, L. C. Malacarne, L. S. Herculano, V. S. Zanuto, M. L. Baesso, and S. E. Bialkowski, Appl. Spectrosc. 67, 1111 (2013).

${ }^{13}$ O. A. Capeloto, G. V. B. Lukasievicz, V. S. Zanuto, L. S. Herculano, N. E. Souza Filho, A. Novatski, L. C. Malacarne, S. E. Bialkowski, M. L. Baesso, and N. G. C. Astrath, Appl. Opt. 53, 7985 (2014).

${ }^{14}$ N. G. C. Astrath, G. V. B. Lukasievicz, L. C. Malacarne, and S. E. Bialkowski, Appl. Phys. Lett. 102, 231902 (2013).

${ }^{15}$ L. Taylor and J. Talghader, Appl. Opt. 54, 9010 (2015).

${ }^{16}$ T. Požar and J. Možina, in Fundamentals of Picoscience, edited by K. D. Sattler (Taylor and Francis, Boca Raton, 2014), p. 553.

${ }^{17}$ G. L. Bennis, R. Vyas, R. Gupta, S. Ang, and W. D. Brown, J. Appl. Phys. 84, 3602 (1998).

${ }^{18}$ J. C. Cheng, L. Wu, and S. Y. Zhang, J. Appl. Phys. 76, 716 (1994).

${ }^{19}$ N. G. C. Astrath, L. C. Malacarne, M. L. Baesso, G. V. B. Lukasievicz, and S. E. Bialkowski, Nat. Commun. 5, 4363 (2014).

${ }^{20}$ O. A. Capeloto, V. S. Zanuto, L. C. Malacarne, M. L. Baesso, G. V. B. Lukasievicz, S. E. Bialkowski, and N. G. C. Astrath, Sci. Rep. 6, 20515 (2016).

${ }^{21}$ J. W. Bray, P. Robinson, D. E. Tyler, and W. T. Black, Metals Handbook: Properties and Selection: Nonferrous Alloys and Special-Purpose Materials (ASM International, 1990), Vol. 2. 
Generation and detection of thermoelastic waves in metals by a photothermal mirror method

TABLE I. Physical parameters of the metals used in numerical calculations [21].

\begin{tabular}{|c|c|c|}
\hline$\overline{\overline{\text { Parameter }}}$ & "Aluminum & $\overline{\text { Copper }}$ \\
\hline$\left(\mathrm{Wm}^{-1} \mathrm{~K}^{-1}\right)$ & 155 & 387 \\
\hline$\rho \quad\left(\mathrm{kgm}^{-3}\right)$ & 2700 & 8887 \\
\hline$C_{p}\left(\mathrm{Jkg}^{-1} \mathrm{~K}^{-1}\right)$ & 1020 & 383 \\
\hline$\alpha_{T}\left(10^{-6} \mathrm{~K}^{-1}\right)$ & 22.8 & 16.7 \\
\hline$E \quad(\mathrm{GPa})$ & 69 & 126 \\
\hline$\nu$ & 0.331 & 0.338 \\
\hline$R$ & 0.92 & 0.61 \\
\hline \multicolumn{3}{|c|}{ Calculated from $\rho, E$, and $\nu$} \\
\hline $\begin{array}{l}c_{\mathrm{P}}\left(\mathrm{ms}^{-1}\right) \\
\end{array}$ & 6165 & 4653 \\
\hline$c_{\mathrm{S}}\left(\mathrm{ms}^{-1}\right)$ & 3098 & 2302 \\
\hline$c_{\mathrm{R}}\left(\mathrm{ms}^{-1}\right)$ & 2888 & 2148 \\
\hline
\end{tabular}

